\title{
Bone Marrow Metastases as an Initial Presentation of Gastrointestinal Adenocarcinoma: A Case Report
}

\author{
Molina-Arrebola María-Angustias ${ }^{1}$, Fernández-Caballero Mariana ${ }^{1}$, Avivar-Oyonarte Cristóbal ${ }^{2}$, \\ Jiménez-Cortés María-Carmen ${ }^{3}$, Hidalgo-Rico María-Angeles ${ }^{4}$ \\ ${ }^{1}$ Department of Biotechnology, Haematology and Haemotherapy Unit, Poniente Hospital, Almería, Spain \\ ${ }^{2}$ Department of Biotechnology, Poniente Hospital, Almería, Spain \\ ${ }^{3}$ Department of Biotechnology, Pathology Unit, Poniente Hospital, Almería, Spain \\ ${ }^{4}$ Department of Internal Medicine, Pulmonology Unit, Poniente Hospital, Almería, Spain
}

Email address:

mariaangustias.molina@ephpo.es (Molina-Arrebola María-Angustias)

\section{To cite this article:}

Molina-Arrebola María-Angustias, Fernández-Caballero Mariana, Avivar-Oyonarte Cristóbal, Jiménez-Cortés María-Carmen, Hidalgo-Rico María-Angeles. Bone Marrow Metastases as an Initial Presentation of Gastrointestinal Adenocarcinoma: a Case Report. Clinical Medicine Research. Vol. 6, No. 4, 2017, pp. 127-130. doi: 10.11648/j.cmr.20170604.12

Received: February 13, 2017; Accepted: April 24, 2017; Published: June 14, 2017

\begin{abstract}
Bone marrow metastases as the initial presentation of a solid tumour are uncommon, especially if the primary neoplasm site cannot be identified during the diagnosis presentation despite laboratory testing and imaging techniques. In these situations, bone marrow examination can lead to a final diagnosis of the primary malignancy. We report an unusual case of osteoblastic metastases from an unknown primary site - this was because no relevant lesions were found from either imaging or endoscopic examination. The patient was sent for a bone marrow biopsy, revealing a morphology replaced by glandular structures and an immunohistochemistry consistent with a gastrointestinal origin. Bone marrow examination was the clue to the final diagnosis and led to specific treatment.
\end{abstract}

Keywords: Bone Marrow, Metastases, Solid Tumour, Immunohistochemistry

\section{Introduction}

Metastases are the most common type of malignant tumour involving bone, and the major primary sites are reported to be breast cancer in women and prostate cancer in men. However, in some cases the primary site cannot be identified by systemic examination and thus the lack of detectable primary neoplasm could delay staging and treatment [1].

Bone marrow examination is a diagnostic tool more commonly used in hematologic malignancies, yet has also been increasingly employed in documenting the metastatic involvement of solid tumours. In these cases, the histological findings such as immunohistochemical and other morphological parameters from the bone biopsy can assess the primary cancer diagnosis. The recognition of metastases in random biopsies is a challenge to haematologists and pathologists alike [2, 3].

\section{Case Report}

A 38-year-old man presented at our hospital with a 2-month history of slight unproductive coughing, profuse sweating and minor weight loss. He also complained of occasional epigastralgia over the previous 7 years with no complementary studies having been performed. He had no personal history of chronic disease and no toxic habits.

Regarding the physical examination, cardiopulmonary auscultation revealed a discrete decrease in vesicular murmur on the left side, with no other relevant findings.

In the laboratory tests, the blood count, renal and liver tests were within normal values. More extensive analysis revealed remarkably high phosphatase activity (1616 UI/L, normal range 48-406 UI/L) as well as elevated carbohydrate antigen 19-9 activity (646 UI/mL, normal range 0-35 UI/mL). The prostate-specific antigen (PSA), however, was normal (Table 1). 
Table 1. Laboratory data.

\begin{tabular}{lll}
\hline Variable & $\begin{array}{l}\text { Patient } \\
\text { data }\end{array}$ & $\begin{array}{l}\text { Reference range in } \\
\text { our hospital, adults }\end{array}$ \\
\hline Hematocrit (\%) & 41 & $36-44$ \\
Haemoglobin (g/dl) & 13.8 & $12-14$ \\
White-cell count (per mm ${ }^{3}$ ) & 11.48 & $4.5-11.5$ \\
Neutrophils (\%) & 79.4 & $40-74$ \\
Lymphocytes (\%) & 13.2 & $19-48$ \\
Monocytes (\%) & 4.0 & $3.4-9$ \\
Eosinophils (\%) & 1.8 & $0-7$ \\
Basophils (\%) & 0.3 & $0-1.5$ \\
Platelet count (per mm $\left.{ }^{3}\right)$ & 175 & $130-450$ \\
Creatinine (mg/dl) & 0.8 & $0.67-1.17$ \\
Aspartate aminotransferase (U/l) & 33 & $0-35$ \\
Alanine aminotransferase (U/1) & 56 & $0-45$ \\
Alkaline phosphatase (U/l) & 1616 & $48-406$ \\
Protein (g/dl) & 7.0 & $6.6-8.3$ \\
Albumin (g/dl) & 4.2 & $3.5-5.2$ \\
Calcium (mg/dl) & 9.3 & $8.8-10.6$ \\
C-reactive protein (mg/dl) & 1.1 & $0.1-0.5$ \\
Alpha-Fetoprotein (ng/ml) & 1.84 & $0-9$ \\
Carbohydrate antigen 19-9 (UI/ml) & 646.1 & $0-35$ \\
Carcinoembryonic antigen (UI/ml) & 5.7 & $0-10$ \\
Prostate specific antigen (ng/ml) & 0.87 & $0-3.1$ \\
\hline
\end{tabular}

A thoracic X-ray was carried out showing a left pleural effusion. Moreover, a routine control with a cranial and pelvic $\mathrm{X}$-ray demonstrated generalized osteoblastic bone metastases. Subsequently, a thoracentesis was carried out and the cytology revealed cells with a reactive mesothelial aspect, together with atypical cell groups of wide cytoplasm, some of them with a vacuolated, hyperchromatic nuclei forming microacinar structures, leading to a suspicion of adenocarcinoma primary neoplasm.

In order to determine the primary site of metastases, a total body tomography scan was performed. However, this failed to uncover any relevant findings except the reported generalized osteoblastic metastases and left pleural effusion. A study of the gastrointestinal tract was performed by gastroduodenoscopy and colonoscopy but both were normal and showed no anomaly.

With no more accessible lesions, the patient was sent for bone marrow biopsy given that pelvic metastases were present. The bone marrow trephine specimen was fixed in Bouin solution, embedded in paraffin and cut into $1.5 \mu \mathrm{m}$ sections, which were stained with haematoxylin and eosin. A microscopic examination revealed the presence of areas of bone marrow replaced by moderately differentiated glandular structures (Figure 1). Immunohistochemistry demonstrated positivity for cytokeratin 7 and cytokeratin 20, and negativity for thyroid transcription factor-1 (TTF1) (Figure 2) and PSA. A negative result for TTF1 ruled out a pulmonary origin and a negative result for PSA ruled out a prostatic origin. With these findings, the most common primary site of metastases was gastrointestinal.

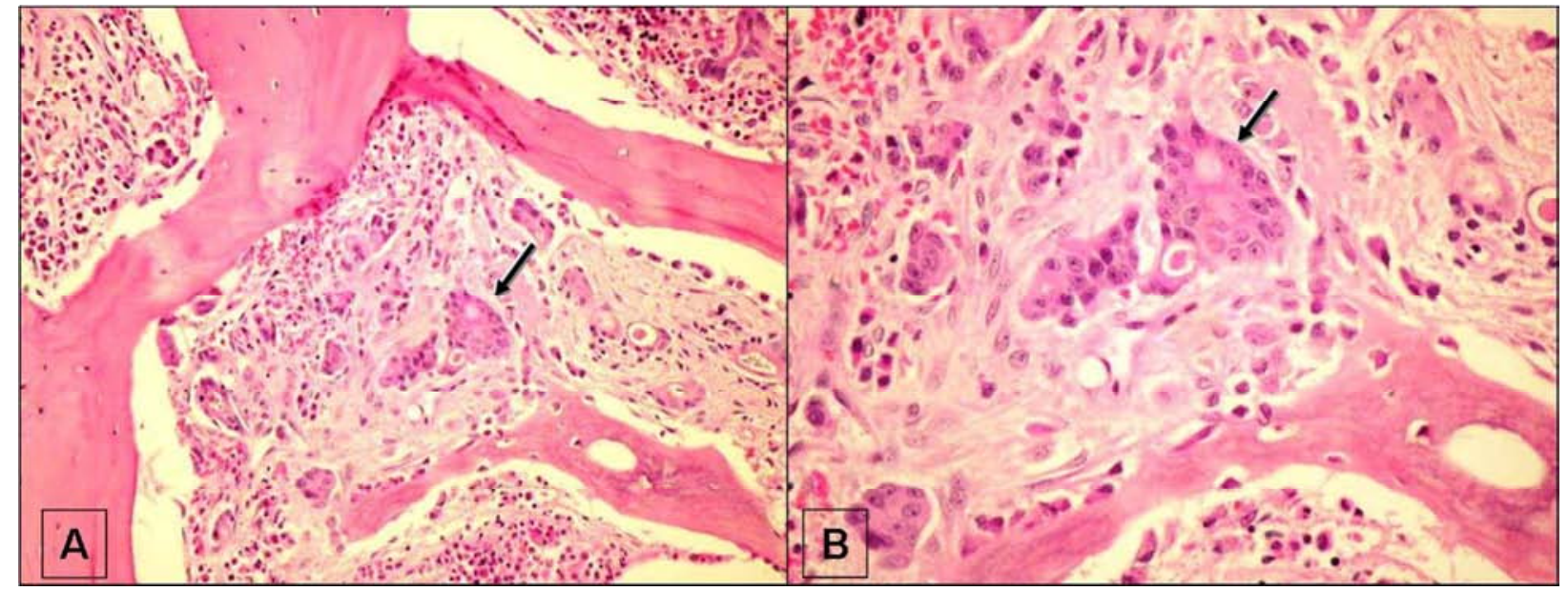

Figure 1. Bone marrow trephine section. Haematoxylin and eosin stain revealed the presence of glandular structures of variable cell shape and size at the intertrabecular level (arrows), consisting of large cytoplasm with round nuclei and nucleoli. Ax20; Bx100.

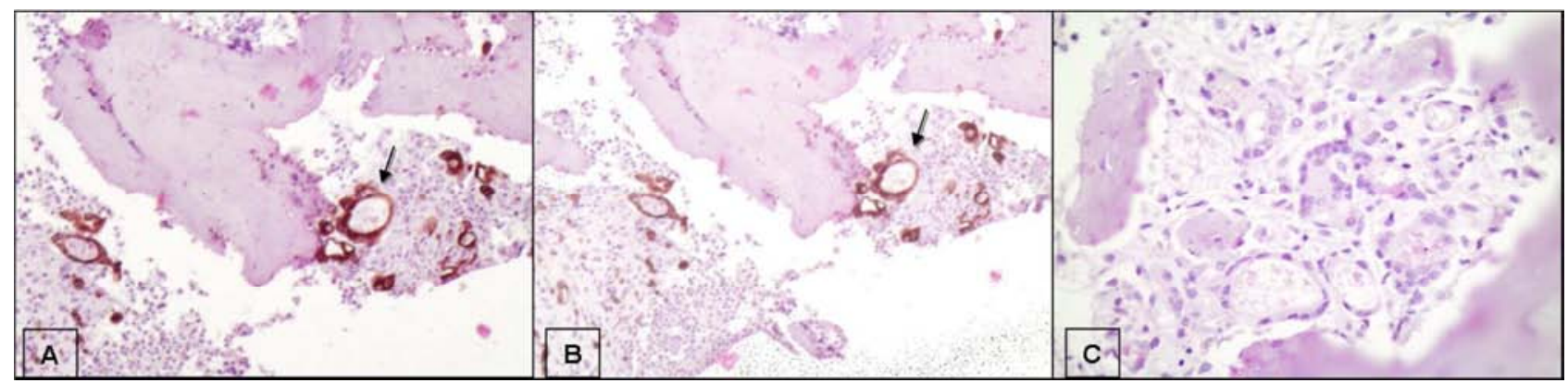

Figure 2. Bone marrow trephine section. Immunohistochemistry demonstrating positivity for cytokeratin 7 (A), cytokeratin 20 (B) (arrows) and negativity for thyroid transcription factor-1 (TTF1) (C). 
Given the suspected diagnosis of gastrointestinal adenocarcinoma with generalized bone metastases, the patient was referred to the Oncology Department. Unfortunately, the disease progressed and he died 2 months later.

\section{Discussion}

Skeletal lesions can be the first manifestation of malignancy in $25-30 \%$ of cases. Breast cancer in women and prostate cancer in men are reported to be the major primary sites of bone metastases, although in the last two decades, lung cancer has also been increasingly reported to be so in both sexes [1, 4]. Imaging studies and diagnostic endoscopic techniques have advanced while new tumour markers have been identified. Despite this, amongst patients with bone metastases, $22-30 \%$ show no evidence of the primary tumour in the diagnosis presentation [5]. In these cases, the study of bone marrow immunohistochemistry and other morphological parameters might reveal the characteristics of the primary origin [6]. Bone marrow involvement from haematological malignancies is commonly found but diagnosing a non-haematological malignancy from bone marrow is rare [7].

Classically, bone marrow obtained by aspiration allows excellent visualization of cell morphology, whereas bone biopsy permits optimal evaluation of cellularity, fibrosis or infiltrative disease. There have been several previous studies pointing to the superiority of bone marrow biopsy over aspirate smears in the diagnosis of metastatic tumours deposits in the marrow are focal and may often elicit a fibrotic response so aspirates may be negative [8]. Moreover, histological sections may allow classification of tumour cell type and immunohistochemistry is used as an adjunct to the morphological findings from the bone marrow; this is of particular value when investigating a patient with a malignancy originating in an unknown primary site.

In some cases, an immunoperoxidase staining for an organ-specific antigen examination might be sufficient to bring out the primary focus. As an example, an immunoperoxidase stain for prostatic acid phosphatase or prostate-specific antigen may be helpful in establishing a diagnosis of metastatic prostate cancer. On the other hand, in a poorly-differentiated tumour, this could be difficult. A leukocyte-common antigen may be used to differentiate lymphohematopoietic neoplasm from other cancers [7].

The study of cytokeratins in primary or metastatic cancer has also been widely used. The positivity of cytokeratin 7 and 20 in metastatic lesions suggests a primary tumour of the gastrointestinal tract or genitourinary tract, as reported: $65-89 \%$ urinary bladder carcinoma, 25-65\% pancreatic cancer, $20-60 \%$ cholangiocarcinoma, $38 \%$ gastric cancer, $30 \%$ thyroid cancer, $10 \%$ lung cancer, $14 \%$ breast cancer, $10 \%$ colon cancer and $8 \%$ prostate cancer [9].

With regard to TTF-1, this is a marker used to identify adenocarcinomas in the distal airway and pulmonary cells as well as for extra pulmonary neuroendocrine cancers. It has been demonstrated that lung cancer cells almost always express TTF-1, so this expression can be used to identify primary or secondary lung cancers [10].

Finally, PSA is a well-recognized tool to elucidate the prostatic origin of metastatic malignancy $[11,12]$.

In the present case, the high level of carbohydrate antigen 19-9 suggested a gastrointestinal neoplasm, as this is usually elevated in pancreatic cancer [13]. On the other hand, the high alkaline phosphatase level put us on the track of bone involvement as this serum marker suggests high bone metabolism and is usually elevated in neoplasm. At this point osteoblastic lesions were found during systemic exploration, and led us to a bone marrow biopsy. The presence of moderately differentiated glandular structures was revealed, consistent with the diagnosis of adenocarcinoma. The PSA study was negative and ruled out a prostatic origin; likewise, the negativity of the TTF-1 ruled out a pulmonary origin. Given the positivity of cytokeratin 7 and 20, a gastrointestinal origin was suggested although the extensive search for the primary site of the adenocarcinoma was unsuccessful.

Despite the limitation of having only a suspected gastrointestinal origin, and the rare initial presentation, there were strong data in the literature that did, indeed, point to a gastrointestinal cause. According to this literature, the gastrointestinal tract is a common site of occult primary tumours $[14,15]$, and adenocarcinoma is the main histological type, accounting for $70 \%$ of all cases, while undifferentiated cancers account for $15 \%$ and squamous cell carcinomas for $10 \%[16]$.

The occurrence of bone marrow metastases has been documented in gastric cancer, usually during the workup for metastatic disease, and relates closely to poorly-differentiated adenocarcinoma with a tendency to occur in those having high lymph node involvement. When bone marrow metastases occur, they are more common in younger patients who have only short-term survival, similar to the case reported $[17,18]$.

On the other hand, colorectal cancer bony metastases are most often associated with synchronous liver and lung metastases. Isolated skeletal metastases are even more uncommon, occurring at an estimated frequency of 1-2\% [19]. With relation to pancreatic cancer, skeletal metastases are uncommon [20], as they are in bile tract and gallbladder localization [21].

\section{Conclusion}

Although marrow metastases can be commonly found in some tumours, diagnosing a non-hematologic malignancy from marrow is a rare event. With no evidence for the primary tumour, the majority of cases follow a short, fatal clinical course with little possibility of employing effective chemotherapy. This example reinforces the relevance of systematic and multidisciplinary differential diagnosis in order to accurately assess the origin and select the most appropriate treatment approach. 


\section{References}

[1] Piccioli A, Maccauro G, Spinelli MS, Biagini R, Rossi B. Bone metastases of unknown origin: epidemiology and principles of management. Journal of Orthopaedics and Traumatology. 2015.

[2] Papac RJ. Bone marrow metastases. A review. Cancer 1994; 74: 2403-13.

[3] Mohanty SK, Dash S. Bone marrow metastasis in solid tumors. Indian J Pathol Microbiol 2003; 46: 613-6.

[4] Piccioli A. Breast cancer bone metastases: an orthopedic emergency. J Orthop Traumatol 2014; 15: 143-4.

[5] Katagiri H, Takahashi M, Inagaki J, Sugiura H, Ito S, Iwata H. Determining the site of the primary cancer in patients with skeletal metastasis of unknown origin: a retrospective study. Cancer 1999; 86: 533-7.

[6] Wedin R, Bauer HC, Skoog L, Söderlund V, Tani E. Cytological diagnosis of skeletal lesions. Fine-needle aspiration biopsy in 110 tumours. J Bone Joint Surg Br 2000; 82: $673-8$.

[7] Ozkalemkas F, Ali R, Ozkocaman V, Ozcelik T, Ozan U, Ozturk H, Kurt E, Evrensel T, Yerci O, Tunali A. The bone marrow aspirate and biopsy in the diagnosis of unsuspected nonhematologic malignancy: a clinical study of 19 cases. BMC Cancer 2005; 5: 144.

[8] Singh G, Krause JR, Breitfeld V. Bone marrow examination: for metastatic tumor: aspirate and biopsy. Cancer 1977; 40: 2317-21.

[9] Chu P, Wu E, Weiss LM. Cytokeratin 7 and cytokeratin 20 expression in epithelial neoplasms: a survey of 435 cases. Mod Pathol 2000; 13: 962-72.

[10] Kitamura H, Yazawa T, Sato H, Okudela K, Shimoyamada H. Small cell lung cancer: significance of RB alterations and TTF-1 expression in its carcinogenesis, phenotype, and biology. Endocr Pathol 2009; 20: 101-7.

[11] Cuzick J, Thorat MA, Andriole G, Brawley OW, Brown PH, Culig Z, Eeles RA, Ford LG, Hamdy FC, Holmberg L, Ilic D,
Key TJ, Vecchia C La, Lilja H, Marberger M, Meyskens FL, Minasian LM, Parker C, Parnes HL, Perner S, et al. Prevention and early detection of prostate cancer. The Lancet Oncology. 2014.

[12] Mostofi FK, Davis CJ, Sesterhenn IA. Pathology of carcinoma of the prostate. Cancer Wiley Subscription Services, Inc., A Wiley Company; 1992; 70: 235-53.

[13] Swords DS, Firpo MA, Scaife CL, Mulvihill SJ. Biomarkers in pancreatic adenocarcinoma: current perspectives. Onco Targets Ther Dove Press; 2016; 9: 7459-67.

[14] Mishra P, Das S, Kar R, Jacob SE, Basu D. Non-haematopoietic malignancies metastasing to the bone marrow: a 5 year record-based descriptive study from a tertiary care centre in South India. Indian J Cancer 2014; 51: 30-4.

[15] Yun HK, Shin MG, Bo D, Kim DW, Cho D, Shin JH, Suh SP, Ryang DW. [Laboratory evaluation of bone marrow metastasis: single institute study]. Korean J Lab Med 2007; 27: 96-101.

[16] Hemminki K, Riihimäki M, Sundquist K, Hemminki A. Site-specific survival rates for cancer of unknown primary according to location of metastases. Int J Cancer 2013; 133: $182-9$.

[17] Chung YS, Choi TY, Ha CY, Kim HM, Lee KJ, Park CH, Fitzpatr LA. An Unusual Case of Osteoblastic Metastasis from Gastric Carcinoma. Yonsei Med J 2002; 43: 377.

[18] Nishidoi H, Koga S. [Clinicopathological study of gastric cancer with bone metastasis]. Gan To Kagaku Ryoho 1987; 14: 1717-22.

[19] Katoh M, Unakami M, Hara M, Fukuchi S. Bone metastasis from colorectal cancer in autopsy cases. J Gastroenterol 1995; 30: 615-8.

[20] Borad MJ, Saadati H, Lakshmipathy A, Campbell E, Hopper P, Jameson G, Von Hoff DD, Saif MW. Skeletal metastases in pancreatic cancer: a retrospective study and review of the literature. Yale J Biol Med 2009; 82: 1-6.

[21] Roa JC, Aretxabala X de, Melo A, Faría G, Araya JC, Villaseca MA, Guzmán P, Roa I. [Detection of bone marrow micrometastases in patients with gallbladder cancer]. Rev Med Chil 2004; 132: 1489-98. 